\title{
Hypothesis for a common-cause underlying both autoimmune-diseases and allergies
}

\author{
Borros M. Arneth \\ Institute of Clinical Chemistry and Laboratory Medicine, \\ University of Technology, \\ Technische Universität - Dresden, \\ Fetscherstr 74, 01307 Dresden, Germany \\ E-mail: borros.arneth@uniklinikum-dresden.de
}

\begin{abstract}
Although autoimmune diseases and allergies have recently been associated with each other, the results of the few studies that are available are contradictory. While some of these studies suggest that there is no evidence to assume a connection between autoimmune diseases and allergies, other studies, including some recent animal studies, suggest a connection between these diseases. In this study, we describe the possible connection between autoimmune diseases and allergies. It is important to note that, for the considerations described here, it will be necessary to consider the two types of diseases in a completely different way than has been done so far.
\end{abstract}

Keywords: autoimmunity; antigen presentation; food allergies; T-lymphocytes; CD4-T-lymphocytes; CD8-T-lymphocytes; allergies.

Reference to this paper should be made as follows: Arneth, B.M. (2011) 'Hypothesis for a common-cause underlying both autoimmune-diseases and allergies', Int. J. Immunological Studies, Vol. 1, No. 3, pp.249-254.

Biographical notes: Borros M. Arneth studied Chemistry/Biochemistry and Medicine, both at the University of Frankfurt/Main, Germany. Since 2001, he has been a Physician and Biochemist. His Diploma thesis was on nuclear magnetic resonance (NMR) spectroscopy and his Medical Doctor thesis was on magnetic resonance (MR) spectroscopy of HIV patients. He is now working as a Research Scientist at the University of Technology in Dresden, Germany.

\section{Introduction}

Recently, several autoimmune diseases have repeatedly been associated with allergies (Lindelof et al., 2008; Valenta et al., 2009; Garn et al., 2007). Unfortunately, the results of the different studies that have investigated this connection have been contradictory. On the one hand, the results of some studies do not allow for a connection to be assumed (Lindelof et al., 2008). On the other hand, recent studies using animal experiments have led to an increased interest in a possible connection between both types of diseases (Valenta et al., 2009; Garn et al., 2007). Here, we describe the ways in which autoimmunity and allergy may be related. In order to understand the considerations described here, it will be necessary to look at the two types of diseases in a completely different manner than has been done so far. 


\subsection{Protein traffic hypothesis}

According to our hypothesis, autoimmune diseases are caused by defects resulting in misguidance in protein trafficking (protein traffic hypothesis) (Arneth, 2008). In addition, defects in apoptosis, programmed cell death and/or defects in other cellular clearance mechanisms could lead to an autosensitisation directed against the body's own cells and proteins.

\subsection{Allergen proteins}

Recently, modern proteomic methods have been used to clarify the identity and structure of some allergen-causing proteins. Surprisingly, intracellular proteins are often identified as allergens. Many of these potential allergens are evolutionarily high-grade conserved proteins, which is interesting, given that similar forms of these proteins are also present within humans.

Under the new postulate proposed here, in which allergies are caused by or at least strongly favoured by, defects in apoptosis, programmed cell death and/or by defects in cellular clearance mechanisms, it becomes understandable that, if such a defect is present, there should be sensitisation against several of the body's own intracellular proteins. These proteins might then be structurally very similar to conserved homologous proteins of other organisms. If an individual comes again into contact with the structurally homologous proteins of other organisms, then an exceedingly robust immune reaction should result.

Of course, the considerations presented here are still hypothetical and need to be investigated in a number of further studies. In a previously described theory, we attributed the emergence of autoimmune diseases to the sensitisation against the body's own cellular proteins, which have been misguided and improperly localised (Arneth, 2008). A number of readers, however, have pointed out several flaws in this hypothesis. First, given the described premises, a larger group of persons should suffer from autoimmune diseases. Second, according to our theory, every human individual should be ill, since cell death happens constitutively in every organism. These critiques have prompted us to reexamine our theory. In addition, some recent studies have provided evidence that supports a connection between autoimmunity and allergy. These studies were aided by the recently developed ability to determine the identity and structure of several allergenic proteins. Thus, intracellular proteins were shown to frequently be responsible for causing both autoimmune diseases and allergies. Moreover, several of these proteins belong, in both cases, to a group of highly evolutionarily conserved proteins, and many of these allergenic proteins occur within humans in a very similar form. In addition, experiments in different animal models have used immunisations with these conserved proteins in order to develop an allergy in the animal. Based on these background studies, we have formulated the following hypothesis: Those patients with allergies are assumed to represent the large group of individuals with 'mild' autoimmune sensitisation, which have been acknowledged to exist by several readers.

As an example, ovalbumin is used as an inducing agent in several animal models of asthma (Garn et al., 2007). Analogously, albumin is also considered to be a major antigen for asthma in humans. 


\section{Evidence}

Many of the currently identified allergens are found as intracellular proteins physiologically. These proteins are highly conserved and are homologous to proteins found in humans (Valenta et al., 2009; Garn et al., 2007; Simon, 2009).

\subsection{Mild and severe damage resulting from the mechanisms of apoptosis}

It seems important to differentiate between slight and severe damage that results from the mechanisms of apoptosis. While slight damage may arise within many humans and may lead to mild disease, severe damage, which involves damage attributable to nuclear degradation, might only rarely occur and may often lead to severe disease. Mild damage resulting from apoptosis should affect the degradation of cytoplasmic proteins and should lead to sensitisation against several intracellular proteins and/or antigens, as is observed in allergies and asthma (Valenta et al., 2009; Garn et al., 2007). However, the degradation of nuclear components should remain uninfluenced in the case of mild apoptotic damage. Severe damage resulting from apoptosis, which leads to errors in nuclear degradation and/or other defects in clearance mechanisms, may, in turn, result in sensitisation against several nuclear components (Herrmann et al., 1998; Schulze et al., 2008). These impaired clearance mechanisms include defects in macrophages and their corresponding low cell counts. Antibodies against nuclear components are frequently found in patients diagnosed with systemic lupus erythematosus (SLE) and other autoimmune diseases (Herrmann et al., 1998; Schulze et al., 2008).

Therefore, it is important to differentiate between mild and severe damage resulting from mechanisms of apoptosis. While slight damage resulting from apoptosis may arise within many otherwise healthy humans and may lead to mild disease, such as asthma and allergies, severe damage resulting from apoptotic mechanisms occur more rarely and should lead to severe disease. Slight apoptotic damage should thus lead to sensitisation against intracellular proteins and/or antigens, as is observed in allergies and asthma. Severe apoptotic damage, in contrast, should lead to sensitisation against nuclear components. Antibodies against nuclear components are frequently found in patients diagnosed with SLE.

Some of the intracellular allergens and self-antigens that also occur in a similar structure within humans that have been described so far include:

1 the actin-binding protein profiling

2 manganese superoxide dismutase (SOD)

3 the leucine zipper DNA-binding protein acidic ribosomal phosphoprotein P2

4 Hom s1 SART-1

5 Hom s3

6 the Hom s4 calcium-binding proteins

7 Hom s5 cytokeratin

$8 \quad \mathrm{P} 75$

9 DNA 
10 RNA

11 the DNA-binding protein $\alpha-N A C$

12 BP230 desmoplakin

13 the Retinal $\mathrm{S}$ antigen arrestin

14 glutamic acid decarboxylase (Valenta et al., 2009)

15 enolases and several calcium-binding proteins, in the case of fungal allergies (Spinozzi et al., 2008; Achatz et al., 1996; Breitenbach et al., 1997; Engel et al., 1997; Unger et al., 1999; Simon-Nobbe et al., 2000)

16 malate dehydrogenase, triose phosphate isomerase and profiling in allergies to watermelon (Pastor et al., 2009) and other fruits.

Some of the extracellular allergens and self-antigens that occur in a similar structure within humans that have been described so far include (c.p., Valenta et al., 2009):

1 ovalbumin and other albumins, as observed in asthma

2 immunglobulins

3 collagen

4 cadherin

5 thyrotropin receptor

6 thyroperoxidase

7 thyroglobulin (Valenta et al., 2009).

In addition, patients that suffer allergies are at risk for the emergence of secondary diseases. Allergies have been associated with an increased incidence of coronary heart disease (CHC, Kounis syndrome) (Sinkiewicz et al., 2008). Allergy sufferers also have a higher predisposition of several arteriosclerotic-caused diseases (Knoflach et al., 2005; Bruneck and Army studies).

We hypothesise that sensitisation against self-antigens and/or proteins represent a substantial risk for the emergence of allergies.

Other hypotheses proposed in order to explain the emergence of allergies include the hygiene hypothesis. This hypothesis states that the decreased contact of babies and infants with environmental antigens alters the negative selection of T-lymphocytes. Therefore, children today grow up under almost 'sterile' conditions, which are frequently thought to be disadvantageous (Valenta et al., 2009; Garn et al., 2007). However, the frequent occurrence of allergies in recent times may have more than one risk factor that might have arisen at the same time. There are also several intriguing questions that arise with respect to the selectivity of allergies in different individuals:

1 Why do some allergies result as a consequence of a particular, single allergen?

2 Why do some individuals develop allergies against multiple, different allergens?

3 If there exists a common cause for all allergies, such as a defect in apoptosis being the basis for all allergies, then how, and why, do different allergies result? 
From the point of view provided here, defects in apoptosis should result in an increased level of immune reactivity. Furthermore, it is necessary to assume that for allergies to emerge, another secondary immunisation seems to be required in addition to this increased reactivity.

Therefore, apoptotic defects can be both genetically transmitted as well as acquired, possibly by viral infection (Mountz et al., 1995). Hence, our modern living conditions would favour both a reduction in the pressure for selection, thereby favouring genetic transmission, as well as also favour viral transmission through which slight apoptotic defects would be tolerated. Since apoptosis at the same time also represents an important mechanism for an organism to fight against viruses, it is quite conceivable that viruses can interact with the mechanisms of apoptosis thereby preventing, or at least inhibiting, cellular death. Viruses could thus ensure their own survival this way.

\section{References}

Achatz, G., Oberkofler, H., Lechenauer, E., Simon, B., Unger, A., Kandler, D., Ebner, C., Prillinger, H., Kraft, D. and Breitenbach, M. (1996) 'Molecular characterization of alternaria alternata and cladosporium herbarum allergens', Adv. Exp. Med. Biol., Vol. 409, pp.157-161.

Arneth, B. (2008) 'A new paradigm in biology - the effect of localisation of a protein on its antigenicity', Iranian Journal of Immunology, Vol. 5, pp.226-228.

Breitenbach, M., Simon, B., Probst, G., Oberkofler, H., Ferreira, F., Briza, P., Achatz, G., Unger, A., Ebner, C., Kraft, C. and Hirschwehr, R. (1997) 'Enolases are highly conserved fungal allergens', Int. Arch. Allergy Immunol., Vol. 113, pp.114-117.

Engel, E., Richter, K., Obermeyer, G., Briza, P., Kungl, A.J., Simon, B., Auer, M., Ebner, C., Rheinberger, H.J., Breitenbach, M. and Ferreira, F. (1997) 'Immunological and biological properties of Bet $\mathrm{v} 4$, a novel birch pollen allergen with two EF-hand calcium-binding domains', J. Biol. Chem., Vol. 272, pp.28630-28637.

Garn, H., Mittermann, I., Valenta, R. and Renz, H. (2007) 'Autosensitization as a pathomechanism in asthma', Ann. N.Y. Acad. Sci., Vol. 1107, pp.417-25.

Herrmann, M., Voll, R.E., Zoller, O.M., Hagendorfer, M., Ponner, B.B. and Kalden, J.R. (1998) 'Impaired phagocytosis of apoptotic cell material by monocyte - derived macrophages from patients with systemic lupus erythematodes', Arthritis Rheum., Vol. 41, pp.1241-1250.

Knoflach, M., Kiechl, S., Mayr, A., Willeit, J., Poewe, W. and Wick, G. (2005) 'Allergic rhinitis, asthma, and atherosclerosis in the Bruneck and army studies', Archives of Internal Medicine, Vol. 165, pp.2521-2526.

Lindelof, B., Granath, F., Tengvall-Linder, M., Lindelof, H. and Ekbom, A. (2008) 'Allergy and autoimmune disease: a registry based study', Clin. Exp. Allergy, Vol. 39, pp.110-115.

Mountz, J.D., Cheng, J., Su, X., Wu, J. and Zhou, T. (1995) 'Autoimmunity, apoptosis defects and retroviruses', Adv. Exp. Med. Biol., Vol. 374, pp.183-201.

Pastor, C., Cuesta- Herranz, J., Cases, B., Perez- Gordo, M., Figueredo, E., de las Heras, M. and Vivanco, F. (2009) 'Identification of major allergens in watermelon', Int. Arch. Allergy Immunol., Vol. 149, pp.291-298.

Schluze, C., Munoz, L.E., Franz, S., Sarter, K., Chaurio, R.A. and Gaipl, U.S. (2008) 'Clearance defieciency - a potential link between infections and autoimmunity', Autoimmun. Rev., Vol. 8 , pp.5-8.

Simon, H.U. (2009) 'Cell death in allergic diseases', Apoptosis, Vol. 14, pp.439-446.

Simon-Nobbe, B., Probst, G., Kajava, A., Oberkofler, H., Susani, M., Crameri, R., Ferreira, F., Ebner, C. and Breitenbach, M. (2000) 'IgE binding epitopes of enolases, a class of highly conserved fungal allergens', J. Allergy Clin. Immunol., Vol. 106, pp.887-895. 
Sinkiewicz, W., Sobański, P. and Bartuzi, Z. (2008) 'Allergic myocardial infarction', Cardiol. J., Vol. 15, pp.220-225.

Spinozzi, F., de Benedictis, D. and de Benedictis, F.M. (2008) 'Apoptosis airway inflammation and anti-asthma therapy: from immunobiology to clinical application', Pediatr. Allergy Immunol., Vol. 19, pp.287-295.

Unger, A., Stöger, P., Simon-Nobbe, B., Susani, M., Crameri, R., Ebner, C., Hintner, H. and Breitenbach, M. (1999) 'Clinical testing of recombinant allergens of the mold alternaria alternata', Int. Arch. Allergy Immunol., Vol. 118, pp.220-221.

Valenta, R., Mittermann, I., Werfel, T., Garn, H. and Renz, H. (2009) 'Linking allergy to autoimmune disease', Trends in Immunology, Vol. 30, pp.109-116. 